\title{
El Impacto de la Educación Ambiental en la Evaluación de la Cultura Ambiental Universitaria: El Caso de la Carrera de Ingeniero Industrial Administrador de la Universidad Autónoma de Nuevo León The Impact of Environmental Literacy on Environmental University Culture Perception: The Case of Industrial Engineering Administrator at the Universidad Autónoma de Nuevo León
}

\author{
Adriana Camacho Gómez ${ }^{1}$, Jorge Omar Moreno Treviño ${ }^{2 *}$, Lilián Angélica Reynosa Martínez ${ }^{3}$ \\ ${ }^{1}$ Universidad Autónoma de Nuevo León, Facultad de Ciencias Químicas, Ave. Universidad S/N, Cd. Universitaria, San \\ Nicolás de los Garza N.L. México, 66455. ORCID: 0000-0002-2712-4797. \\ ${ }^{2}$ Universidad Autónoma de Nuevo León, Facultad de Economía, Av. Lázaro Cárdenas 4600, Monterrey N.L. México, \\ 64930. ORCID: 0000-0002-5658-6763 \\ ${ }^{3}$ Universidad Autónoma de Nuevo León, Facultad de Ciencias Químicas, Ave. Universidad S/N, Cd. Universitaria, San \\ Nicolás de los Garza N.L. México, 66455. ORCID: 0000-0002-9391-6127. \\ *Autor de correspondencia: Jorge.morenotr@uanl.edu.mx \\ DOI: https://doi.org/10.29105/revig1.1-9
}

\begin{abstract}
This paper studies the impact of the learning unit (UA) "Environment and Sustainability" on the perception of the university's environmental culture using the Industrial Engineering Administrator (IIA) students of the Autonomous University of Nuevo León (UANL) as a case study. For this purpose, we developed a survey with a representative stratified statistical design by gender and semesters of the IIA career, with an exploratory-descriptive nature, and with a quantitative-qualitative approach. The final sample is 620 students, 313 men, and 307 women, chosen at random from the objective population. The questionnaire comprises more than 100 items organized into 40 questions with information on the student's context, socioeconomic dimensions, as well as on their recycling consumption, and their perception of environmental culture. The results show there is homogeneity in the response of a high percentage of questions between students who have attended the UA and those who have not. However, there is statistical evidence that shows that having taken the UA makes students stricter in their evaluation and perception of the environmental culture within the Faculty, significantly reducing the positive evaluation and increasing its negative evaluation. These results are robust for two types of econometric models considered in the present study.
\end{abstract}

Keywords:

Environmental literacy, sustainability, environmental culture perception, college students.

\begin{abstract}
Resumen
El presente artículo estudia el impacto de la unidad de aprendizaje (UA) "Ambiente y Sustentabilidad" sobre la percepción de cultura ambiental universitaria usando como caso de análisis: Estudiantes de Ingeniero Industrial Administrador (IIA) de la Universidad Autónoma de Nuevo León (UANL). Para este fin se realizó un estudio con diseño estadístico estratificado representativo por género en todos los semestres que conforman la carrera de IIA, con carácter exploratorio-descriptivo, y con un enfoque cuantitativo-cualitativo. La muestra está constituida por 620 estudiantes, 313 hombres y 307 mujeres, elegidos aleatoriamente. El cuestionario comprende más de 100 reactivos, con información en dimensiones de contexto del estudiante, socioeconómico de su hogar, hábitos de reciclaje, consumo, uso de materiales reciclables, y su percepción sobre cultura ambiental. Los resultados muestran, entre otros aspectos, que existe una homogeneidad en la respuesta de un alto porcentaje de reactivos entre estudiantes que han cursado La UA "Ambiente y Sustentabilidad", y aquellos que no. Sin embargo, existe evidencia estadística que demuestra que haber cursado la UA hace que los estudiantes sean más estrictos en su evaluación y percepción de la cultura ambiental dentro de la Facultad, siendo este resultado robusto para dos tipos de modelos econométricos considerados en el presente estudio.
\end{abstract}

Palabras clave:

Cultura ambiental, sustentabilidad, percepción de cultura ambiental, estudiantes universitarios. 


\section{Introducción}

En la actualidad los seres humanos afrontamos la problemática ambiental como una crisis que cada vez es más evidente; la deforestación, el consumo abusivo, la extinción masiva de las especies, la contaminación ambiental, éstos son solo algunos de los principales problemas medioambientales que existen hoy en día. La comunidad educativa ha realizado diversos esfuerzos para detener el crecimiento de esta problemática, se ha optado por incluir actividades o Unidades de aprendizaje sobre la concientización ambiental en las Instituciones de Educación Superior (IES), con la finalidad de generar una cultura ambiental en los futuros profesionistas, promoviendo el respeto al medio ambiente con la conciencia de que el futuro ambiental del planeta depende de toda la humanidad.

Como sociedad, debemos comprender y reconocer la existencia de límites al desarrollo social, al crecimiento económico y a la explotación de los ecosistemas. Es por lo que se debe replantear nuestra vinculación con el entorno natural mediante un desarrollo sustentable. Aunque las Universidades han hecho acercamientos hacia la educación ambiental, actualmente se requiere algún modelo de evaluación de éstas que contemple el contexto de los Objetivos de Desarrollo Sustentable de acuerdo con la ONU, incluyendo una autoevaluación integral que considere las "tres esferas de sustentabilidad": económica, social y ambiental. (Bohne, et al. 2019).

Reconociendo que las organizaciones universitarias son la fuente principal en generar conocimientos, bienes intangibles, formar profesionales y transformar la realidad de su entorno; deben contribuir a la generación del conocimiento científico y tecnológico, sirviendo al sector productivo y de la sociedad de la información, considerando el impacto que las organizaciones de educación superior poseen sobre la sociedad, de tal forma que contribuyan al progreso y desarrollo a través de la gestión del conocimiento. (Montilla, 2018)

En México, gracias a la importancia que se le da a la Educación verde, diferentes IES decidieron desarrollar las políticas para la sustentabilidad, unidades de aprendizaje, y grupos de estudiantes enfocados a la sustentabilidad, como actividades que adoptarían diferentes nombres y nomenclaturas de acuerdo con la estructura de cada universidad. Entre las experiencias pioneras se encuentran el Programa Ambiental Universitario de la Universidad Autónoma de Baja California (UABC), la Agenda Ambiental de la Universidad Autónoma de San Luis Potosí (UASLP), el Programa de Medio Ambiente de la Universidad Nacional Autónoma de México (UNAM) y el de la Universidad de Guadalajara (UDEG); a éstas se fueron sumando un creciente número de IES tan distintas como distantes en el territorio mexicano, que alimentaron con abundante experiencia la implementación de políticas para la sustentabilidad.(Martínez, 2015)

La UANL Introduce dentro de sus Unidades de Aprendizaje de Formación General Universitaria; la UA "Ambiente y Sustentabilidad", a partir del plan 2006 se incorpora al programa de estudios de la carrera de IIA, con el propósito de incorporar el enfoque humanístico y profesional hacia el ambiente y el desarrollo sustentable en la estructura curricular de los estudios de Licenciatura de la UANL, con el propósito de que los estudiantes asuman su formación conscientes de desarrollar su capacidad profesional y de participación en las propuestas de solución de la problemática ambiental a nivel local, regional y global y adquieran a la vez conciencia, valores y actitudes indispensables para la generación de una cultura ambiental, comprometidos en la consolidación del desarrollo sustentable mediante el ejercicio de su profesión.

Esta UA es impartida en el quinto semestre, y sus temas principales son:

- Reconocimiento del funcionamiento de la naturaleza a través del estudio y comprensión holística de los principios básicos de la ecología.

- Identificación de la problemática ambiental mediante el análisis de actividades antropogénicas que generan contaminantes al ambiente, que sobrepasan el umbral de tolerancia de los ecosistemas

- Relación entre la contaminación ambiental y la salud de los seres vivos a través del reconocimiento de las propiedades de emisiones al ambiente y su relación a la integridad de los ecosistemas y la salud humana.

- Comprensión de los principios de sustentabilidad analizando el concepto de desarrollo sustentable y sus implicaciones en acuerdos y convenios entre países para la mitigación de la problemática ambiental a nivel globa y

- El análisis de la política ambiental, promoción de la conservación y sustentabilidad del ambiente a partir del conocimiento de la legislación y normatividad en materia ambiental. (Alfaro, et al. 2009).

\section{Revisión de Literatura}

La Educación Ambiental (EA) es definida como el conjunto de actitudes, intenciones de comportamiento y conocimientos ambientales que posee una persona (Kibert, 2000).

La percepción ambiental la definen como un proceso psicológico relevante en la relación individuo medio ambiente que conlleva a la formación de una conciencia ambiental orientada hacia la sostenibilidad (Alea y Jaula, 2006).

Con respecto a la sustentabilidad, este es un concepto que va más allá del tema ambiental, pues se puede relacionar con el 
desarrollo y futuro de las organizaciones y su capacidad de trascender y mantenerse en óptimo funcionamiento a lo largo del tiempo; las organizaciones universitarias, así como el entorno donde estas se desenvuelven no escapan de esta realidad. (Montilla, 2018). También se define como un proyecto a largo plazo donde los procesos deben ser sostenibles, robustos, resilientes y adaptativos (Calvente, 2007). Las IES son esenciales como fuerza de cambio que afectan el bienestar humano y natural, además se habla sobre los indicadores más populares, que evalúan la integración de la sustentabilidad en las IES. Identificando a STARS como el sistema más completo de evaluación, GreenMetric tiene categorías de energía y cambio climático, desperdicios, transporte y agua, y por último COMPLEXUS en la sustentabilidad social y académica, dejando en segundo plano la categoría de infraestructura. También se encontró que específicamente en las IES de México, la Universidad Nacional Autónoma de México (UNAM) utiliza GreenMetric para evaluar su educación verde, y la Universidad de Monterrey (UDEM) utiliza STARS como sistema de evaluación, mientras que la Universidad de Guanajuato utiliza COMPLEXUS. (Mendoza, 2016)

Diferentes Universidades, comprometidas con la problemática ambiental se han dado a la tarea de analizar los resultados de las acciones implementadas para desarrollar una cultura ambiental efectiva en sus estudiantes. En la Universidad de Córdova se evaluó el nivel de conciencia ambiental de estudiantes de la, encontrando como resultado que los alumnos tienen un déficit alto en cuestiones ambientales (información), una baja participación real en iniciativas relacionadas con el tema, pero con un alta participación potencial interpretando que en ocasiones podemos estar motivados para actuar, pero el entorno no ofrece esa alternativa, por otro lado podemos tener la capacidad de actuar adecuadamente, pero no estar interesados (Gomera, 2008). El concepto de "techo de cristal" es incluido en el estudio de (González, et al. 2015) dirigido a los procesos de sustentabilidad universitaria, haciendo una fuerte crítica sobre las barreras sociales, económicas y académicas que han imposibilitado avances reales y significativos, llegando a la conclusión de que las universidades Iberoamericanas deben de construir colectivamente la sustentabilidad de la comunidad universitaria, mediante una movilización interna, y que una de sus misiones debe ser formar ciudadanos que puedan influir en otras comunidades. Existe un escepticismo sobre el desarrollo de sustentabilidad en la India que la mantiene en atraso a nivel global, causando que el conocimiento y desarrollo de este sea cada vez más caro y difícil de obtener. Las Instituciones Educativas carecen de información sobre el tema y la literatura existente proviene del extranjero, por tal razón no existen planes adecuados que integren el tema de sustentabilidad. (Jose, 2016). En el Tecnológico de Matamoros se realizó un estudio donde los resultados muestran un impacto de la materia Desarrollo Sustentable, sobre la conciencia ambiental de sus estudiantes y sugieren que la EA sea implementada desde el nivel básico incluyendo bases científicas. (Vargas, et al. 2017). En la Universidad de Sonora, México; se implementó un proceso de formación de sentidos en tres dimensiones: "mejorar el medio natural", "generar conciencia en las personas acerca de la relación ser humano-naturaleza" e "impactar en las relaciones ser humano-naturaleza de las generaciones futuras" con resultados favorables, ya que éstos tienen gran impacto en el actuar en pro del medio ambiente natural, social y humano. (Hernández, et al. 2017). En la búsqueda de establecer la relación de la sustentabilidad con las universidades, se demostró que el mismo desarrollo de la sustentabilidad obliga a las universidades a evolucionar para transmitir conocimientos del tema a través del uso de las tecnologías de la información y la interacción con el entorno. (Montilla, 2018).

\section{Metodología}

Los datos que se presentan en este trabajo de investigación proceden de una encuesta elaborada para analizar los hábitos de cultura ecológica, utilizando una versión propia a partir del cuestionario de mayor referencia (Kibert, 2000). Esta versión permitió adaptar a un solo instrumento las dimensiones de información necesarias para el estudio y así ser aplicado a los estudiantes de la carrera de Ingeniero Industrial Administrador (IIA) de la Facultad de Ciencias Químicas (FCQ), de la Universidad Autónoma de Nuevo León (UANL).

El marco de diseño de muestra se creó considerando como sujeto de estudio al estudiante universitario representativo en la población total de IIA en la UANL. Para este fin, se procedió a diseñar una muestra irrestricta aleatoria estratificada en dos dimensiones: semestre de carrea (10 semestres) y género (masculino y femenino), dando un total de 20 estratos de clasificación.

De esta forma, se tomó como base los datos de la composición estudiantil de la FCQ-UANL usados en (Camacho, et al. 2018), y actualizando para el nuevo ciclo escolar 2018. Por tanto, se consideró como población objetivo la descomposición observada en el Tabla 1 a continuación:

Usando un criterio de población irrestricta aleatoria auto-ponderada, la fórmula para cada uno de los estratos génerosemestre considerado fue basada en el criterio definido por la Ec. 1 a continuación: 


$$
n_{j}(\alpha, p, d)=\left[\frac{N_{j} Z_{\alpha}^{2} p(1-p)}{d_{2}\left(N_{j}-1\right)+N_{j} Z_{\alpha}^{2} p(1-p)}\right]
$$

Ec. 1

En este caso, $n_{j}(\alpha, p, d)$ indica el tamaño de muestra necesario para alcanzar un nivel de confianza estadística $\alpha$, dado un parámetro poblacional de proporción "p", y un nivel de tolerancia "d", es función de los parámetros anteriores, el tamaño de población contenido en el estrato de interés $N_{j}$ y el valor de la normal estándar evaluado en el nivel de confianza estadística $Z_{\alpha}$. 
Tabla 1.

Composición de Población Objetivo:

Estudiantes de la carrera IIA, FCQ-UANL

\begin{tabular}{|c|c|c|c|}
\hline Semestre & Mujeres & Hombres & Total \\
\hline 1 & 224 & 273 & 497 \\
\hline 2 & 117 & 109 & 226 \\
\hline 3 & 224 & 224 & 448 \\
\hline 4 & 70 & 83 & 153 \\
\hline 5 & 166 & 171 & 337 \\
\hline 6 & 60 & 78 & 138 \\
\hline 7 & 145 & 148 & 293 \\
\hline 8 & 55 & 51 & 106 \\
\hline 9 & 107 & 113 & 220 \\
\hline 10 & 62 & 80 & 142 \\
\hline Total & $\mathbf{1 2 3 0}$ & $\mathbf{1 3 3 0}$ & $\mathbf{2 5 6 0}$ \\
\hline
\end{tabular}

Fuente: Censo estudiantil de semestre agosto-diciembre 2018 en carrera IIA, FCQ-UANL (2018).

En un estudio previo dado por (Herrera, et al. 2016), se encontró que un muy alto porcentaje de estudiantes universitarios en la edad relevante consideran que proteger el medio ambiente es necesario e importante, así como una alta preocupación por el deterioro del medio ambiente.

Utilizando un criterio conservador de $95 \%$ de importancia en los ítems anteriores ${ }^{1}$ para determinar el porcentaje poblacional base sobre la relevancia de la cultura ambiental nos permita calcular el tamaño crítica de muestra, en combinación con un criterio $\alpha=0.05$ y un nivel de tolerancia al error de $d=0.06$, el tamaño de muestra requerido es de 552 encuestas distribuidas a lo largo de los 20 estratos considerados, siendo 274 para mujeres y 278 para hombres. Para este caso, decidimos aplicar varios cuestionarios auxiliares por estrato, lo anterior para controlar por la potencial tasa de no respuesta y el error en la respuesta por parte de los estudiantes al contestar su encuesta. Si consideramos que esa no respuesta en los cuestionarios fue aleatoria entre los alumnos de todos los estratos considerados, la muestra final que tenemos es de un total de 620 cuestionarios distribuidos entre los distintos estratos tal y como se presenta en el Tabla 2.

De esta forma, a partir de los los criterios estadísticos anteriores, la muestra posee al menos una precisión de $6.0 \%$ (admitiendo incluso reducirse a 5.5\%) y un error estadístico con límite superior en $5.0 \%$. La aplicación de encuestas se realizó el mes de octubre, durante el semestre agosto-diciembre 2018. Se informó a los estudiantes encuestados del objetivo del estudio, así mismo, se explicó la metodología para el llenado de esta; además se comentó que su información tendría un tratamiento anónimo. Los alumnos participaron en este ejercicio por decisión voluntaria. El tiempo de respuesta osciló entre 10 a 15 minutos como máximo. Al término de cada aplicación se llevó un control para completar la muestra representativa por semestre y género. La captura se realizó al finalizar la aplicación de las encuestas por parte de los investigadores, así se pudo conformar la base de datos final cuyo análisis se realizó usando el paquete estadístico STATA (Versión 15) por brindar un amplio conjunto de herramientas de análisis econométrico.

Con respecto a la variable de impacto considerada en nuestro trabajo definida por la UA "Ambiente y Sustentabilidad" (AyS), al constituir una materia obligatoria esta representa estrictamente en econometría y estadística "un instrumento", esto es, una variable que no está correlacionada con alguna decisión del estudiante, que es obligatoria para todos los alumnos a partir del $5^{\circ}$ semestre, y que por tanto, no está estadísticamente correlacionada con el contexto socioeconómico de cada estudiante, permitiendo contar con dos grupos: un grupo control (sin haber cursado la materia) y un grupo experimental (el grupo de estudiantes que ya cursaron la materia).

\footnotetext{
${ }^{1}$ Las preguntas relevantes son para este estimador "Considero importante conocer la manera de proteger el Medio Ambiente" 97.1\%, "Me preocupan mucho los efectos del deterioro medioambiental en mi calidad de vida y la de mi familia" 96.2\%". En este caso, se utilizará un criterio conservador de $95 \%$.
} 
Tabla 2.

Composición de muestra por diseño irrestricto aleatorio estratificado:

Alumnos encuestados por semestre, genero

\begin{tabular}{|c|c|c|c|}
\hline $\begin{array}{c}\text { Semestre } \\
\text { Encuestado }\end{array}$ & Hombres & Mujeres & Total \\
\hline $\mathbf{1}$ & 36 & 36 & 72 \\
\hline $\mathbf{2}$ & 31 & 30 & 61 \\
\hline $\mathbf{3}$ & 34 & 33 & 67 \\
\hline $\mathbf{4}$ & 30 & 31 & 61 \\
\hline $\mathbf{5}$ & 35 & 34 & 69 \\
\hline $\mathbf{6}$ & 27 & 28 & 55 \\
\hline $\mathbf{7}$ & 33 & 32 & 65 \\
\hline $\mathbf{8}$ & 28 & 26 & 54 \\
\hline $\mathbf{9}$ & 34 & 31 & 65 \\
\hline $\mathbf{1 0}$ & 25 & 26 & 51 \\
\hline Total: & $\mathbf{3 1 3}$ & $\mathbf{3 0 7}$ & $\mathbf{6 2 0}$ \\
\hline
\end{tabular}

Fuente: Elaboración propia usando cuestionario "Encuesta de Hábitos y Cultura Ecológica (2018)”.

Nuestro objetivo es medir la percepción de la calidad educativa en la facultad para un estudiante "i” dada por una variable $P_{i}$ es diferente si este cursó la materia "AyS" o no la ha cursado (dado respectivamente por una variable dicotómica $D_{i}=1$ y $D_{i}=0$ respectivamente). Por tanto la hipótesis nula correspondiente $H_{o} \mathrm{y}$ la hipótesis alternativa $H_{1}$ correspondientes son que la percepción esperada de un estudiante con y sin haber cursado la materia, contrastado con que sean diferentes:

$$
\begin{array}{lll}
H_{o}: & E\left[P_{i} \mid D_{i}=1\right]=E\left[P_{i} \mid D_{i}=0\right] & \text { Ec. } 3 \\
H_{1}: & E\left[P_{i} \mid D_{i}=1\right] \neq E\left[P_{i} \mid D_{i}=0\right] &
\end{array}
$$

En particular, dada a la naturaleza el análisis de resultados se realiza obteniendo la media condicional en haber o no tomado el curso, para posteriormente realizar una prueba simple de diferencia en medias de Satterwhite con varianzas diferenciadas entre sexos, usando un criterio estadístico de "t" de Satterwhite, y obteniendo el nivel de significancia estadística de la prueba de diferencias (valor-p) en cada caso con $v=\left[\frac{\left(\frac{s_{1}^{2}}{n_{1}}+\frac{s_{0}^{2}}{n_{0}}\right)^{2}}{\left(\frac{s_{1}^{2}}{n_{1}}\right)^{2}}+\frac{\left(\frac{s_{0}^{2}}{n_{0}}\right)^{2}}{\left(n_{1}-1\right)}\right]$ definiendo los grados de libertad correspondientes a la hipótesis. En este caso, la prueba estadística simple correspondiente bajo la premisa de diferentes varianzas desconocidas y estimadas en cada población está en función de los promedios observados $\bar{P}_{1}, \bar{P}_{0}$ y las desviaciones estándar observadas $S_{P_{1}}, S_{P_{0}}$ para cada grupo por:

$$
\frac{\Delta P}{S_{\Delta P}}=\frac{\overline{P_{1}}-\bar{P}_{0}}{\sqrt{\frac{S_{P_{1}}}{n_{1}}-\frac{S_{P_{0}}}{n_{0}}}} \sqsupset t_{\alpha, v}
$$

Donde la hipótesis de igualdad de medias en percepción se rechaza si el valor calculado de "t" a partir de los datos es superior a un nivel dado por una tolerancia estadística $t_{\alpha,\left(n_{1}+n_{2}-1\right)}$.

Finalmente, a manera de controlar por otros factores observados, se estimó un modelo economérico probabilístico 
condicional (Probit) definiendo algunas variables de contexto de cada estudiante para medir si la probabilidad de evaluar la calidad de la cultura ambiental en la facultad es afectada por cursar o no la materia. En este caso, el modelo probit condicional se define como:

$$
\begin{array}{lr}
\operatorname{prob}\left[P_{i}=K \mid X_{i}, D_{i}, \varepsilon_{i}\right]=\Phi\left(X_{i}, D_{i}, \varepsilon_{i}\right) & \text { Ec. } 5 \\
\operatorname{prob}\left[Y_{i}^{*}>0 \mid X_{i}, D_{i}, \varepsilon_{i}\right]=\Phi\left(X_{i}, D_{i}, \varepsilon_{i}\right) & \text { Ec. } 6 \\
Y^{*}{ }_{i}=\beta X_{i}+\delta D_{i}+\varepsilon_{i} & \text { Ec. } 7 \\
\varepsilon_{i} \sqcap N\left(0, \sigma_{\varepsilon}{ }^{2}\right) & \text { Ec. } 8
\end{array}
$$

Donde $Y^{*}{ }_{i}$ es una variable latente con un vector de determinantes de contexto dados por $X_{i}, D_{i}$ y el término no observado $\varepsilon_{i}$ se distribuye normal estándar, lo cual define por tanto la probabilidad de que la percepción de la calidad de cultura sea $P_{i}=K$, donde " $\mathrm{K}$ " es el nivel de calidad en la cultura educativa (Buena. Mediana, y Mala). Este modelo se estima por máxima verosimilitud y permite medir el efecto de estudiar la UA de "AyS" sobre la percepción de calidad educativa a través de la significancia estadística del coeficiente " $\delta$ " ponderado por la función matemática adecuada que representa el normal estándar acumulada $\Phi(z)$ en un valor “z” determinado (Greene, 2017).

\section{Resultados}

La evidencia de este estudio y sus respectivas pruebas estadísticas se concentran en dos aspectos: primero mostrar las diferencias entre hombres y mujeres de la muestra en la Tabla 3a y los semestres de primer a quinto semestre (grupo control) y compararlos con respecto a los estudiantes de sexto semestre en adelante (grupo experimental) en la Tabla 3b.

La Tabla 3a muestra que las variables que son estadísticamente diferentes entre hombres y mujeres son la probabilidad de trabajar (siendo casi 8 porciento mayor para los hombres, relativo a las mujeres), mientras que las mujeres poseen un mayor número de carros promedio en su casa, pero tienden a viajar más personas en el automóvil con respecto a los hombres.

En términos de percepción ambiental, ya entrando en materia cuando comparamos estudiantes que ya cursaron y no han cursado la materia relevante a cultura ambiental, observamos que como es de esperarse, los estudiantes que ya cursaron la UA de "AyS" estadísticamente tienen mayor edad (2.53 años), tienen una mayor probabilidad de trabajar (45.41 por ciento), pertenecen a hogares con menos miembros (-0.1760 personas), utilizan más el automóvil para desplazarse a la escuela (14.15 por ciento) y finalmente viajan más solos en el automóvil por viaje realizado (-0.5109 personas).

Por otra parte, y ya entrando en materia de evaluación de percepción de cultura ambiental, la Tabla 4 mide la diferencia en la respuesta a la percepción de la cultura ambiental tanto en los estudiantes como el nivel de cultura ambiental de la facultad. Un primer resultado muestra que no existe diferencia significativa entre la percepción de la cultura ambiental de los estudiantes para con respecto a los mismos estudiantes, con y sin materia, ya que la diferencia observada no es estadísticamente significativa. También observamos que el porcentaje de estudiantes que declaran que el nivel de cultura ambiental de la facultad "Bueno" no es estadísticamente diferente entre estudiantes con y sin haber cursado la materia, el cual ronda entre el 25.61 y 26.94 por ciento respectivamente: esto es, aproximadamente 1 de cada 4 estudiantes en la facultad, considera que la cultura ambiental en la misma es Buena, sin diferencia entre haber cursado la materia "AyS" o no. Sin embargo, los resultados cambian dramáticamente en las otras dos respuestas. En particular, observamos que la respuesta sobre nivel de cultura ambiental "Regular" se reduce al cursar la materia, al pasar de 71.21 por ciento, a 63.37 por ciento, significando una reducción estadísticamente significativa en este nivel de percepción. Lo que, es más, y como era de esperarse, el nivel de cultura ambiental evaluada en "Mala" se incrementó posterior a cursar la UA de "AyS" en los estudiantes al pasar de 4.48 porciento, a 10.73 porciento, un incremento significativo de 5.88 porciento.

Estos resultados no deben interpretarse de manera negativa, sino como el resultado natural de cualquier proceso educativo: el estudiante que descubre alternativas de reciclaje y cuidado del ambiente, desea que éstas se implementen y de no hacerlo, castiga con su "voto" la falta de asertividad en las políticas ambientales.

Finalmente, con el objetivo de tener un estudio robusto sobre el resultado anterior, se procedió a estimar tres modelos Probit, condicionando la respuesta en semestre, edad y género, esto es, tratando de medir el impacto puro de haber cursado la materia eliminando el potencial efecto correlacional de estos cofactores. La Tabla 5 muestra tres columnas, donde en cada una se estima un modelo probit para cada nivel de percepción de nivel de cultura ambiental en la facultad: Buena, Regular y Mala, respectivamente. Los resultados son robustos, concluyentes y muy interesantes. En este caso, ninguno de 
los cofactores analizados pareciera modificar la probabilidad de evaluar la calidad ambiental de la facultad en los niveles "Bueno" o "Regular", sin embargo, estos resultados se revierten cuando evaluamos la probabilidad de respuesta "Mala". En particular, interpretando los coeficientes como "cambios en probabilidad" siguiendo la metodología clásica de (Greene, 2017) encontramos que estudiantes de semestres superiores tienen a reducir la probabilidad de evaluar la calidad ambiental como "Mala" en 12.66 por ciento, estudiantes de mayor edad tienden a evaluar peor la calidad ambiental al incrementar por cada año la probabilidad de contestar esa evaluación en 21.27 por ciento, además, no existe una diferencia significativa entre la respuesta de evaluación "Mala" asociada a género, esto es, hombres y mujeres no afectan la probabilidad de respuesta. Sin embargo, como resultado principal y destacado, encontramos que el hecho de haber cursado la UA definida por "AyS" incrementa la probabilidad de contestar que la cultura ambiental de la facultad es "Mala" en 52.94 porciento, siendo este resultado estadísticamente significativo. Esto es, uno de cada dos estudiantes que cursan la asignatura cambia su percepción de la cultura ambiental de la facultad a mala, lo cual equivale a decir que se vuelve mucho más exigente con las políticas ambientales y la cultura que prevalece en su entorno universitario inmediato.

Todos los resultados anteriores deben leerse con una óptica propositiva: el hecho de que la unidad de aprendizaje concientice al estudiante sobre la importancia del medio ambiente debe ser acompañada de propuestas concretas que de la mano de los estudiantes, por ejemplo a través de sus representantes en sus distintas carreras, conviertan esa preocupación en actividades específicas encaminadas a fortalecer el nivel de cultura ambiental, particularmente porque otros resultados derivados de la misma encuesta muestran que los estudiantes que ya cursaron "AyS" perciben el problema de la contaminación como uno complejo en donde todos somos parte del mismo, y su solución por tanto requiere de políticas integrales para resolverse. ${ }^{2}$

En conclusión, los estudiantes universitarios de la carrera IIA si cambian su percepción de cultura ambiental en su entorno inmediato, particularmente en su facultad, al cursar la UA de "Ambiente y Sustentabilidad", y los resultados sugieren que tienden a ser más exigentes con la manera en que esta cultura ambiental se implementa en su entorno, dando origen a la necesidad de buscar en conjunto con ellos soluciones a la problemática ambiental desde la misma facultad, aprovechando su interés y compromiso por este tema.

\footnotetext{
${ }^{2}$ Estos resultados que escapan al propósito del presente artículo se exploraran en dos artículos próximos a someterse derivados de la misma encuesta.
} 
Tabla 3a.

Medias y diferencias en medias para variables socioeconómicas, por sexo del estudiante

\begin{tabular}{lcccc}
\hline Variable & Hombres & Mujeres & Diferencia & \\
\hline Edad (en años) & 19.869 & 19.844 & 0.0253 & \\
Trabaja $(1=\mathrm{Si}, 0=\mathrm{No})$ & 0.4952 & 0.4137 & 0.0815 & {$[\mathrm{~b}]$} \\
Alumno foráneo $(1=\mathrm{Si} 0=\mathrm{No})$ & 0.0897 & 0.0847 & 0.0051 & \\
Trabaja padre $(1=\mathrm{Si} 0=\mathrm{No})$ & 0.9612 & 0.9639 & -0.0028 & \\
Trabaja madre $(1=\mathrm{Si} 0=\mathrm{No})$ & 0.5273 & 0.5490 & -0.0217 & \\
Totalde miembros en el hogar & 3.9761 & 3.9030 & 0.0731 & \\
Usa coche para ir a la escuela (1=Si 0=No) & 0.4452 & 0.5085 & -0.0632 & \\
Cuantos coches tienes en casa (Número) & 2.3533 & 2.1614 & 0.1919 & {$[\mathrm{~b}]$} \\
Cuantas personas viajan en tu coche (Número) & 1.7484 & 1.9884 & -0.2400 & {$[\mathrm{~b}]$} \\
Ingreso total del hogar declarado (pesos mensuales) & $\$ 17,298.1$ & $\$ 21,822.7$ & $-\$ 4524.6$ & \\
\hline Tamaño de muestra: $\mathrm{n}=620$ & 313 & 307 & & \\
\hline
\end{tabular}

Notas:

1) Cada columna presenta la media muestral de cada variable condicional a cada categoría de sexo del estudiante, y la diferencia de medias.

2) La tercera columna muestra la diferencia de medias entre sexo de estudiantes, el error estándar correspondiente a la prueba (" $t$ " de Student) de diferencias con varianza desconocidas y diferentes se muestra entre paréntesis inferior al valor correspondiente.

$3)$ En variables del tipo dicotómico $(1=\mathrm{Si} 0=\mathrm{No})$, la interpretación del promedio es porcentaje de respuesta declarada: por ejemplo 0.78 se interpreta como $78 \%$ de respuesta afirmativa.

4) Los indicadores de significancia de la prueba estadística (valor-p) son: [a] $\mathrm{p}<0.10$, [b] $\mathrm{p}<0.05$, [c] $\mathrm{p}<0.01$, [d] $\mathrm{p}<0.001$. Las áreas sombreadas en cada tabla son estadísticamente significativas.

Fuente: Estimaciones propias usando cuestionario Encuesta de Hábitos y Cultura Ecológica (2018).

Tabla 3b.

Medias y diferencias en medias para variables socioeconómicas, por control de haber cursado ó no "AyS"

\begin{tabular}{lcccc}
\hline Variable & $\begin{array}{c}\text { Con } \\
\text { "AyS" }\end{array}$ & Sin "AyS" & $\begin{array}{c}\text { Diferencia } \\
\text { (Con - Sin) }\end{array}$ \\
\hline Edad (en años) & 21.2071 & 18.6702 & 2.5372 & {$[\mathrm{~d}]$} \\
Trabaja $(1=\mathrm{Si}, 0=\mathrm{No})$ & 0.6966 & 0.2424 & 0.4541 & {$[\mathrm{~d}]$} \\
Alumno foráneo $(1=\mathrm{Si} 0=\mathrm{No})$ & 0.0965 & 0.0790 & 0.0175 & \\
Trabaja padre $(1=\mathrm{Si} 0=\mathrm{No})$ & 0.9652 & 0.9602 & 0.0049 & \\
Trabaja madre $(1=\mathrm{Si} 0=\mathrm{No})$ & 0.5087 & 0.5636 & -0.0549 & \\
Total de miembros en el hogar & 3.8472 & 4.0232 & -0.1760 & {$[\mathrm{~b}]$} \\
Usa coche para ir a la escuela $(1=\mathrm{Si} 0=\mathrm{No})$ & 0.5527 & 0.4112 & 0.1415 & {$[\mathrm{~d}]$} \\
Cuantos coches tienes en casa (Número) & 2.3165 & 2.2085 & 0.1081 & \\
Cuantas personas viajan en tu coche (Número) & 1.6257 & 2.1366 & -0.5109 & {$[\mathrm{~d}]$} \\
Ingreso total del hogar declarado (pesos mensuales) & 31050.3 & 37403.4 & -6353.1 & \\
\hline Tamaño de muestra: $\mathrm{n}=620$ & 290 & 330 & & \\
\hline
\end{tabular}

Notas:

1) Cada columna presenta la media muestral de cada variable condicional a cada categoría de sexo del estudiante, y la diferencia de medias.

2) La tercera columna muestra la diferencia de medias entre sexo de estudiantes, el error estándar correspondiente a la prueba (" $t$ ” de Student) de diferencias con varianza desconocidas y diferentes se muestra entre paréntesis inferior al valor correspondiente.

3) En variables del tipo dicotómico $(1=\mathrm{Si} 0=\mathrm{No})$, la interpretación del promedio es porcentaje de respuesta declarada: por ejemplo 0.78 se interpreta como $78 \%$ de respuesta afirmativa.

4) Los indicadores de significancia de la prueba estadística (valor-p) son: [a] p <0.10, [b] p <0.05, [c] p $<0.01$, [d] p $<0.001$. Las áreas sombreadas en cada tabla son estadísticamente significativas.

Fuente: Estimaciones propias usando cuestionario Encuesta de Hábitos y Cultura Ecológica (2018). 
Tabla 4.

Medias y diferencias en medias para variables de hábitos y percepción de cultura ambiental en FCQ, UANL

\begin{tabular}{|c|c|c|c|c|}
\hline Variable & $\begin{array}{l}\text { Con } \\
\text { "AyS" }\end{array}$ & $\begin{array}{l}\text { Sin } \\
\text { "AyS" }\end{array}$ & $\begin{array}{l}\text { Diferencia } \\
\text { (Con - Sin) }\end{array}$ & \\
\hline $\begin{array}{l}\text { Observa cultura ambiental en sus compañeros de facultad } \\
(1=\text { Si } 0=\text { No) }\end{array}$ & 0.6069 & 0.6455 & -0.0386 & \\
\hline $\begin{array}{c}\text { Nivel de cultura ambiental de la facultad }(F C Q) \\
\text { BUENA } \quad(1=\mathrm{Si} 0=\mathrm{No})\end{array}$ & 0.2561 & 0.2394 & 0.0166 & \\
\hline $\begin{array}{ll}\text { REGULAR } & (1=\mathrm{Si} 0=\mathrm{No}) \\
\text { MALA } & (1=\mathrm{Si} 0=\mathrm{No})\end{array}$ & $\begin{array}{l}0.6367 \\
0.1073\end{array}$ & $\begin{array}{c}0.7121 \\
0.04848\end{array}$ & $\begin{array}{c}-0.0754 \\
0.0588\end{array}$ & $\begin{array}{l}{[\mathrm{b}]} \\
{[\mathrm{c}]}\end{array}$ \\
\hline Tamaño de muestra: $n=620$ & 290 & 330 & & \\
\hline
\end{tabular}

Notas:

1) Cada columna presenta la media muestral de cada variable condicional a cada categoría de sexo del estudiante, y la diferencia de medias.

2) La tercera columna muestra la diferencia de medias entre sexo de estudiantes, el error estándar correspondiente a la prueba (" $t$ " de Student) de diferencias con varianza desconocidas y diferentes se muestra entre paréntesis inferior al valor correspondiente.

$3)$ En variables del tipo dicotómico $(1=\mathrm{Si} 0=\mathrm{No})$, la interpretación del promedio es porcentaje de respuesta declarada: por ejemplo 0.78 se interpreta como $78 \%$ de respuesta afirmativa.

4) Los indicadores de significancia de la prueba estadística (valor-p) son: [a] $\mathrm{p}<0.10,[\mathrm{~b}] \mathrm{p}<0.05$, [c] p $<0.01$, [d] p $<0.001$. Las áreas sombreadas en cada tabla son estadísticamente significativas.

Fuente: Estimaciones propias usando cuestionario Encuesta de Hábitos y Cultura Ecológica (2018).

Tabla 5.

Modelo simple de probabilidad (Probit): evaluación del nivel de cultura ambiental en la FCQ-UANL.

\begin{tabular}{|c|c|c|c|c|}
\hline \multirow[b]{2}{*}{ Variable de control } & \multicolumn{4}{|c|}{$\begin{array}{l}\text { Respuesta de Nivel de Cultura } \\
\text { en la Facultad de Ciencias Químicas }\end{array}$} \\
\hline & Buena & Regular & Mala & \\
\hline Semestre del alumno & $\begin{array}{c}0.0101 \\
(0.0486)\end{array}$ & $\begin{array}{c}0.0552 \\
(0.0445)\end{array}$ & $\begin{array}{l}-0.1266 \\
(0.0615)\end{array}$ & [b] \\
\hline Edad (en años) & $\begin{array}{l}-0.0617 \\
(0.0631)\end{array}$ & $\begin{array}{l}-0.0577 \\
(0.0541)\end{array}$ & $\begin{array}{c}0.2127 \\
(0.0642)\end{array}$ & [d] \\
\hline Género $(1=$ Hombre $0=$ Mujer $)$ & $\begin{array}{c}0.1197 \\
(0.1101)\end{array}$ & $\begin{array}{l}-0.0619 \\
(0.1051)\end{array}$ & $\begin{array}{l}-0.0999 \\
(0.1541)\end{array}$ & \\
\hline $\begin{array}{l}\text { Cursó "Ambiente y Sustentabilidad" } \\
(1=\mathrm{Si} 0=\mathrm{No})\end{array}$ & $\begin{array}{c}0.1595 \\
(0.2208)\end{array}$ & $\begin{array}{l}-0.3391 \\
(0.2123)\end{array}$ & $\begin{array}{c}0.5294 \\
(0.3163)\end{array}$ & [a] \\
\hline Constante & $\begin{array}{c}0.3496 \\
(1.0979)\end{array}$ & $\begin{array}{c}1.5043 \\
(0.9488)\end{array}$ & $\begin{array}{l}-5.2501 \\
(1.1598)\end{array}$ & [d] \\
\hline Tamaño de muestra: & 619 & 619 & 619 & \\
\hline
\end{tabular}

Notas:

1) Cada columna es un modelo probabilístico Probit asociado a la respuesta positiva al nivel de percepción sobre la evaluación del nivel de cultura ambiental.

2) Cada coeficiente debe interpretarse como el cambio en la probabilidad de dar esa respuesta: un coeficiente de 0.20 implica que aumenta la probabilidad de dar esa respuesta en $20 \%$.

3) Los errores estándar de la estimación por máxima verosimilitud están reportados entre paréntesis

4) Los indicadores de significancia de la prueba estadística (valor- $p$ ) son: [a] $\mathrm{p}<0.10,[\mathrm{~b}] \mathrm{p}<0.05$, [c] p <0.01, [d] p <0.001. Las áreas sombreadas en cada tabla son estadísticamente significativas.

Fuente: Estimaciones propias usando cuestionario Encuesta de Hábitos y Cultura Ecológica (2018). 


\section{Conclusiones}

Este artículo responde una pregunta sencilla pero importante desde la perspectiva de la planeación ambiental de una institución universitaria: ¿impacta la educación ambiental a la visualización, percepción y evaluación de los estudiantes sobre la calidad ambiental de su entorno educativo inmediato? Nuestros resultados sugieren que la respuesta es afirmativa para el caso de los estudiantes de la carrera Ingeniero Industrial Administrador en la Facultad de Ciencias Químicas de la Universidad Autónoma de Nuevo León.

En este trabajo se utiliza una metodología de estrategia de muestreo estadístico que permite construir una muestra representativa para los estudiantes de esta carrera como población objetivo, considerando 20 estratos diferenciados entre los distintos sexos (mujeres y hombres) y semestres (10 a 10o semestre) en los cuales los estudiantes cursan su carrera universitaria. Además, aprovechando que la Unidad de Aprendizaje de "Ambiente y Sustentabilidad" es obligatoria para todos los estudiantes de quinto semestre (y que por tanto, todo estudiante de semestre superior ya la ha cursado), es posible contar con un instrumento econométrico, esto es, una variable pura que no está correlacionada con el género, nivel de ingreso, o contexto del estudiante, pero que podría permitir medir un efecto en el cambio de la percepción y hábitos de cultura ambiental antes y después de cursar dicha materia. Para este fin, usando como instrumento dicha UA, se compararon los promedios de distintas métricas de percepción en calidad ambiental de los estudiantes entre un grupo "control" (sin haber cursado la materia) y "experimental" (ya ha cursado la materia), empleando la técnica de pruebas de igualdad medias bajo poblaciones distintas con varianza desconocida ("t" de Satterwhite) y un modelo probabilístico condicional (probit) para controlar el efecto de otros cofactores como edad, semestre y género e identificar así el impacto puro de haber cursado la materia de "Ambiente y Sustentabilidad".

Entre otros resultados, el análisis de este artículo muestra que, en general, posterior a cursar la UA de "Ambiente y Sustentabilidad" los estudiantes se vuelven mucho más estrictos en la evaluación de la cultura ambiental de la facultad, en particular reduciendo la evaluación regular e incrementando la evaluación negativa, mostrando que el conocer los criterios de hábitos como reciclaje y uso de mejores estrategias de cuidado del medio ambiente transforman al estudiante en uno más consciente, pero también más exigente con su entorno inmediato, siendo en este caso, la facultad.

Lo anterior permite deducir que las instituciones educativas más próximas al estudiante (en este caso las facultades donde el estudiante se desempeña) aún puede hacer una labor para mejorar su percepción para con los alumnos, y mostrar que ambos (estudiantes e institución) persiguen los mismos fines y comparten los mismos principios en términos de la importancia que tiene la cultura ambiental, y por tanto, están de acuerdo a las políticas implementadas para fortalecer su correcta operación en la cotidianidad del ambiente universitario.

En conclusión, la provisión adecuada, constante, y eficiente de servicios de recolección y reciclaje de basura, de la mano de otra políticas internas que pudieran ser costo-efectivas para la propia facultad como campañas de reforestación, rehabilitación de parques y cuidado del medio ambiente, puede fortalecer la percepción de cultura hacia dentro de la Facultad, alineando la calidad educativa ambiental a las expectativas que los estudiantes tienen de su ejercicio práctico en su entorno universitario diario e inmediato.

\section{Agradecimientos}

Los autores agradecen el trabajo de Brisa Carolina Garza Guel, Monserrat Guerrero Mendoza, y Cinthya Vanessa Sánchez Niño, estudiantes de la carrera de Ingeniero Industrial Administrador de la Facultad de Ciencias Químicas UANL, quienes colaboraron como asistentes de investigación en la realización del presente proyecto.

\section{Referencias}

Alea, A., and Jaula, J. La percepción ambiental en estudiantes de la Universidad de Pinar del Río. noviembre 18, 2020, de Innovación Educativa, Instituto Politécnico Nacional México Sitio web: https://www.redalyc.org/pdf/1794/179421187004.pdf, 2006.

Alfaro, J. Limón, B. Martínez, G. and Tijerina, G. Ambiente y Sustentabilidad. Por una educación ambiental. México. Grupo Editorial Patria, 2009.

Bohne, A., Bruckmann, M., y Martínez, A. El desarrollo sustentable en las instituciones de educación superior: un verdadero desafío. noviembre 19, 2020, de Revista Digital Universitaria Sitio web: http://www.revista.unam.mx/wp-content/uploads/v20_n5_a3_Eldesarrollo-sustentable-en-las-instituciones-de-educaci\%C3\%B3n-superior-un-verdadero-desaf\%C3\%ADo.pdf, 2019.

Calvente, A. El concepto moderno de sustentabilidad. noviembre 18, 2020, de UAIS Sustentabilidad Sitio web: http://www.sustentabilidad.uai.edu.ar/pdf/sde/UAIS-SDS-100-002\%20-\%20Sustentabilidad.pdf, 2007.

Camacho, A., Moreno J.O., y Reynosa, L. Usos de tiempo y TICs en estudiantes universitarios: evidencia de la carrera de IIA en la UANL. En Construcción social de una cultura digital educativa: SOMECE 2018. Editorial: Sociedad Mexicana de Computo en la Educación., p.p. 1255-1265, 2018. 
Camacho, A., Moreno J.O., y Reynosa, L. Encuesta de Hábitos y Cultura Ecológica, el caso de los estudiantes de los estudiantes de Ingeniero Industrial Administrador, de la Facultad de Ciencias Quimicas UANL. 2018.

Gomera, A. La conciencia ambiental como herramienta para la educación ambiental: Conclusiones y reflexiones de un estudio en el ámbito universitario. noviembre 19, 2020, de web de la Universidad de Córdoba Sitio web: http://www.uco.es/servicios/dgppa/sepa, 2008.

González, E., Meira-Cartea, P., y Martínez, C. Sustentabilidad y Universidad: retos, ritos y posibles rutas. noviembre 18, 2020, de SCIELA-Revista de la educación superior Sitio web: http://www.scielo.org.mx/scielo.php?script=sci_arttext\&pid=S0185$27602015000300004,2015$.

Greene, W. Econometric Analysis (7 $7^{\text {th }}$ Edition). Prentice-Hall. 2017.

Hernández, A., Zayas, F., y Camarena, B. La experiencia universitaria en la formación de sentidos acerca de la sustentabilidad. noviembre 18, 2020, de Revista Internacional de Cooperación \& Desarrollo Sitio web: http://revistas.usbbog.edu.co/index.php/Cooperacion/article/view/3115/2605, 2017.

Herrera, K., Acuña, M., Ramírez, M., y De la Hoz, M. Actitud y conducta pro-ecológica de jóvenes universitarios. Red de Revistas Científicas de América Latina y el Caribe, España y Portugal; vol. 32, no. 13, pp. 456-477, 2016.

Jose, PD. Sustainability Education in Indian Business Schools: A Status Review. November 18, 2020, de SCIELO - Sustainability Education in Indian Business Schools Sitio web: http://www.scielo.org.co/scielo.php?script=sci_arttext\&pid=S169202792016000100015\&lang=es, 2016.

Kibert, N. An Analysis Of The Correlations Between The Attitude, Behavior, And Knowledge Components Of Environmental Literacy In Undergraduate University Students. November 18, 2020, de CiteSeerX Sitio web: http://citeseerx.ist.psu.edu/viewdoc/summary?doi=10.1.1.26.2627, 2000.

Martínez, C., y González, E. Las políticas para la sustentabilidad de las Instituciones de Educación Superior en México: entre el debate y la acción. noviembre 18, 2020, de Revista de la educación superior Sitio web: http://www.scielo.org.mx/scielo.php?script=sci_arttext\&pid=S0185-27602015000200004, 2015.

Mendoza, Y. Sistemas de evaluación de la sustentabilidad en las Instituciones de Educación Superior. noviembre 18, 2020, de SCIELO Sitio web: http://www.scielo.org.mx/scielo.php?script=sci_arttext\&pid=S2007-78582016000200065, 2016.

Montilla, A. Vinculación entre Sustentabilidad y Organizaciones Universitarias. noviembre 19, 2020, de Revista Científica Electrónica de Ciencias Gerenciales / Scientific e-journal of Management Science Sitio web: www.revistanegotium.org.ve / núm. 39 (año 13) pág. 46-65, 2018.

Vargas, C., Rosario, R., y Briones, C. Impacto de la materia Desarrollo Sustentable en el Cambio de la Conciencia Ambiental de los Estudiantes del Nivel Superior. noviembre 18, 2020, de Revista Luna Azul Sitio web: https://www.redalyc.org/pdf/3217/321753629002.pdf, 2017. 